\title{
Closed-form solution to an economic growth logistic model with constant migration
}

\section{Solução fechada para um modelo de crescimento econômico logístico com migração constante}

\author{
João Plínio Juchem Neto ${ }^{1}$, Julio Cesar Ruiz Claeyssen², Daniele Ritelli \\ e Giovanni Mingari Scarpello ${ }^{3}$ \\ ${ }^{1}$ Universidade Federal do Pampa, RS, Brasil \\ plinio@unipampa.edu.br \\ ${ }^{2}$ Universidade Federal do Rio Grande do Sul, RS, Brasil \\ julio@mat.ufrgs.br \\ ${ }^{3}$ Università di Bologna, Itália \\ daniele.ritelli@unibo.it; giovannimingari@libero.it
}

\begin{abstract}
This paper considers a Solow-Swan economic growth model with the labor force ruled by the logistic equation added by a constant migration rate, I. We prove the global asymptotic stability of the capital and production per capita. Considering a Cobb-Douglas production function, we show this model to have a closed-form solution, which is expressed in terms of the Beta and Appell $F_{1}$ special functions. We also show, through simulations, that if $I>0$, it implies in a smaller capital and product per capita in the short term, but in a higher capital and product per capita in the middle and long terms. In both cases, these per capita variables converge to the same steady-state given by the model without migration. If I $<0$ the transient behavior is the opposite. Finally, if $I=0$, we recover the solution for the pure logistic case, involving Gauss' Hypergeometric Function ${ }_{2} F_{1}$.
\end{abstract}

Keywords: Solow economic growth model, Logistic labor growth, Migration, Beta function, Appell function.

\section{Resumo}

Neste trabalho consideramos o modelo de crescimento econômico de Solow-Swan com a mão-de-obra governada pela equação logística adicionada por uma taxa de migração constante, I. Provamos a estabilidade assintótica global do capital e produto per capita. Considerando uma função de produção de Cobb-Douglas, mostramos que este modelo admite uma solução fechada, expressa em termos das funções especiais Beta e de Appel $F_{1}$. Também mostramos, através de simulações, que quando I $>0$, o modelo apresenta um menor nível de capital e produto per capita no curto prazo; mas apresenta um maior nível de capital e produto per capita no médio e longo prazos. Em ambos os casos, estas variáveis per capita convergem para o mesmo estado de equilíbrio do modelo sem migração. Se $I<0$, o comportamento transiente é o oposto. Finalmente, se $I=0$, então recuperamos a solução do modelo logístico puro, envolvendo a função hipergeométrica de Gauss ${ }_{2} F_{1}$.

Palavras-chave: Modelo de crescimento econômico de Solow, Crescimento logístico da mão-de-obra, Migração, Função Beta, Função de Appell. 


\section{Introduction}

The main assumption of the Solow-Swan model for economic growth (Solow, 1956; Swan, 1956) is that labor force obeys the Malthusian Law. Adding to it a CobbDouglas production function, the resulting model has a well known analytical solution. But such an unbounded exponential rise is unrealistic in some cases, implying an overestimation of the real economic growth: therefore recent works have replaced the Malthusian Law by increasing but bounded population growth models. Donghan (1998) proposed the replacement of the Malthusian Law by the Verhulst (logistic) Law in the Solow-Swan model, without actually solving it, but proving the reaching of a steady state. In the same paper, he proves a comparison, a limit and a stability theorem for the capital per capita evolution, assuming a strictly increasing and bounded labor force. Following Donghan (1998), Mingari Scarpello e Ritelli (2003) showed that the Solow-Swan model with a logistic population law has a closed-form solution in terms of the Gauss' Hypergeometric function ${ }_{2} F_{1}$. Maldonado e Brida (2007) considered afterwards the Von Bertalanffy Law which also gave raise to a closed-form solution involving ${ }_{2} F_{1}$, and Accinelli e Brida (2005) introduced the Richards Law (a generalized logistic model). Juchem Neto e Claeyssen (2015a) also considered a logistic population law in a Spatial Solow Model, and in a recent work (Juchem Neto et al., 2015b), showed that the addition of a constant migration rate in the standard Solow model (Solow, 1956; Swan, 1956), with an exponentially growing labor force (Malthusian Law), admits a closed-form solution in terms of the Gauss' Hypergeometric function ${ }_{2} F_{1}$.

Following the methodology presented in Juchem Neto et al. (2015b), in this work we consider a constant migration rate $I$ in the extended model proposed by Mingari Scarpello e Ritelli (2003), which considers a logistic growth for the labor force, and show that this model has a closed-form solution in terms of the Beta and $F_{1}$ Appell's functions, provided $I>-\frac{\alpha^{2}}{4 \beta}$. In the following section we review the Solow-SwanVerhulst Model with Cobb-Douglas production, and in section 3 we discuss the stability and steady-state of the capital and output per capita, and solve the model modified by a constant migration term. In section 4 we carry out some simulations for a set of parameter and discuss the results. Finally, section 5 provides our conclusions.

\section{The Solow model with a logistic labor force growth}

Mingari Scarpello e Ritelli (2003) have shown that the Solow-Swan growth model, considering a Cobb-Douglas production function and a logistic growth law for the labor force, has a closed-form solution in terms of the Gauss' Hypergeometric function ${ }_{2} F_{1}$. The production function considered by them was:

$$
Y=A L(t) k^{\varphi}(t),
$$

where $A>0$ is a constant technological parameter, $\varphi \in$ $(0,1)$ is the output elasticity of capital ( $\varphi$ closer to 0 means a labor intensive economy, and $\varphi$ closer to 1 a capital intensive one), and:

$$
L(t)=\frac{\alpha L_{0} e^{\alpha t}}{\alpha+\beta L_{0}\left(e^{\alpha t}-1\right)}
$$

is the labor force at time $t$, which is solution of the Verhulst (logistic) equation:

$$
\dot{L}=L(\alpha-\beta L), L(0)=L_{0}>0 .
$$

Here, $\alpha>0$ is called the intrinsic growth rate, $\beta>0$ the crowding coefficient, and:

$$
K=\lim _{t \rightarrow \infty} L(t)=\frac{\alpha}{\beta}
$$

is the environment's carrying capacity, that is, the maximum labor force that the economy can hold. In addition, they supposed $\alpha>\beta$, and implicitly $L_{0}<K$, in order to have an asymptotically increasing, and bounded from above, population.

The capital per capita, $k(t)$, is solution of the Bernoulli ordinary differential equation:

$$
\left\{\begin{array}{l}
\dot{k}+(n(t)+\delta) k=s A k^{\varphi} \\
k(0)=k_{0}
\end{array}\right.
$$

where:

$$
n(t)=\frac{\dot{L}(t)}{L(t)}
$$

is the labor force growth rate, $s \in(0,1)$ the constant saving rate, $\delta \in(0,1)$ the constant depreciation rate, and $k_{0}>0$ the initial capital per capita. The assumption that $L(t)$ is given by (2), implies that:

$$
\begin{aligned}
& k(t)=\left\{\alpha^{\varphi-1}\left[\alpha-\beta L_{0}\left(1-e^{\alpha t}\right)\right]^{1-\varphi} e^{(\varphi-1)(\alpha+\delta) t} \times\right. \\
& \left.\times\left[k_{0}^{1-\varphi}+s(1-\varphi) A \alpha^{-\varphi}\left(\alpha-\beta L_{0}\right)^{\varphi-1}\left[\Im_{t}-\Im_{0}\right]\right]\right\}^{\frac{1}{1-\varphi}},
\end{aligned}
$$


where:

$\mathfrak{I}_{t}=\frac{\alpha e^{(1-\varphi)(\alpha+\delta) t}}{(1-\varphi)(\alpha+\delta)}{ }_{2} \mathrm{~F}_{1}\left(\begin{array}{c|c}1-\varphi, \frac{\alpha}{(1-\varphi)(\alpha+\delta)} & \frac{\beta L_{0}}{\beta-\alpha} e^{\alpha t} \\ \frac{\alpha}{(1-\varphi)(\alpha+\delta)}+1 & \beta,\end{array}\right.$

and:

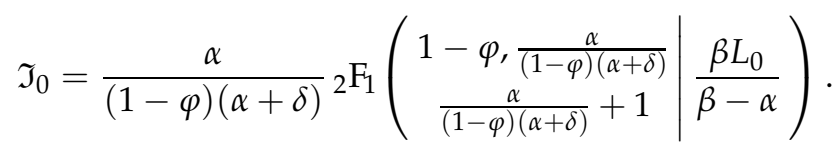

Note that ${ }_{2} \mathrm{~F}_{1}\left(\begin{array}{c}a_{1}, a_{2} \\ a_{3}\end{array} \mid z\right)$ is the Gauss' Hypergeometric Function, and its definition and detailed properties can be found in Erdélyi (1953) and Rainville (1960). A quick overview is presented in Boucekkine e Ruiz-Tamarit (2008).

\section{The Solow model with a logistic labor force and constant migration}

Now we add to the dynamics of the labor force (3) a constant migration rate $I$ so that the differential equation governing the evolution of $L$ becomes:

$$
\dot{L}=L(\alpha-\beta L)+I,
$$

where we still assume $\alpha>\beta$. The solution of the above first order differential equation is given by (Banks, 1994; Matis e Kiffe, 2004):

$$
L(t)=\frac{1}{2 \beta}\left[\alpha+b \frac{\left(1+c e^{-b t}\right)}{\left(1-c e^{-b t}\right)}\right]
$$

where:

$$
a=\frac{2 \beta L_{0}-\alpha}{b}, b=\sqrt{\alpha^{2}+4 \beta I}, c=\frac{a-1}{a+1} .
$$

By (10), we must have at least $I \geq-\frac{\alpha^{2}}{4 \beta}$, to guarantee $b \in \mathbb{R}$. The carrying capacity $K$ relevant to (9) is given by:

$$
K=\lim _{t \rightarrow \infty} L(t)=\frac{\alpha+b}{2 \beta},
$$

and we will consider $L_{0}<K=\frac{\alpha+b}{2 \beta}$. Notice that, when $I=0$, we recover the solution of the pure logistic equation (2).

If $I>0$, then $b>\alpha$ by (10), and we have a greater carrying capacity than the case without migration (4); if $-\frac{\alpha^{2}}{4 \beta} \leq I<0$, then $b<\alpha$, and we verify a lower carrying capacity than the case with no migration $(b=\alpha)$.

Considering (9) and the change of variable ${ }^{1}$ :

$$
z=\bar{k}^{1-\varphi}
$$

\footnotetext{
${ }^{1}$ From now on, the over score indicates variables concerning the model with migration.
}

we can rewrite (5) as the following linear differential equation, now in $z(t)$ :

$$
\dot{z}-(\varphi-1)(\delta+\bar{n}(t)) z=(1-\varphi) s A,
$$

subject to the initial condition $z(0)=z_{0}=\bar{k}_{0}^{1-\varphi}$. Defining the integrating factor:

$$
\begin{aligned}
H(t) & =(\varphi-1) \int_{0}^{t}(\delta+\bar{n}(\tau)) \mathrm{d} \tau \\
& =(\varphi-1) \delta t+(\varphi-1) \ln \left(\frac{L(t)}{L_{0}}\right),
\end{aligned}
$$

we have that the solution of (13) is given by:

$$
z(t)=z_{0} e^{H(t)}+(1-\varphi) s A e^{H(t)} \int_{0}^{t} e^{-H(\tau)} \mathrm{d} \tau .
$$

\section{Stability and Steady-State}

Before computing an analytical expression for (15), and therefore for $\bar{k}(t)$, let us discuss stability and asymptotes of $\bar{k}(t)$.

\section{Proposition 1.}

i. The capital per capita $\bar{k}(t)$ is globally asymptotically stable for $I \in\left[L_{0}\left(\beta L_{0}-\alpha\right),+\infty\right)$.

ii. The capital per capita goes to infinity at a finite time if $I \in\left(-\frac{\alpha^{2}}{4 \beta}, L_{0}\left(\beta L_{0}-\alpha\right)\right)$, that is, $\lim _{t \rightarrow t^{*}} \bar{k}(t)=+\infty$ where $t^{*}=\frac{1}{b} \ln \left(\frac{\alpha-b}{\alpha+b} c\right)$.

\section{Proof.}

i. We will show that $z(t)$ is globally asymptotically stable in the interval $I \in\left[L_{0}\left(\beta L_{0}-\alpha\right),+\infty\right)$. Then, by the continuity of the change of variable (12), we conclude that $\bar{k}(t)$ is also globally asymptotically stable.

First, let us consider $v(t)$ a solution of (13) subjected to the arbitrary positive initial condition $v(0)=v_{0}>0$. This solution is given by:

$$
v(t)=v_{0} e^{H(t)}+(1-\varphi) s A e^{H(t)} \int_{0}^{t} e^{-H(\tau)} \mathrm{d} \tau .
$$

Considering (15) and (16), we have that

$$
|z(t)-v(t)|=\left|z_{0}-v_{0}\right| e^{H(t)} .
$$

By (9), and for $I \in\left[L_{0}\left(\beta L_{0}-\alpha\right),+\infty\right)$, we have that $L(t) \rightarrow+\infty$ as $t \rightarrow+\infty$. Besides of that, because $\varphi \in$ $(0,1), \lim _{t \rightarrow+\infty} H(t)=-\infty$ by (14). Therefore:

$$
\lim _{t \rightarrow+\infty}|z(t)-v(t)|=0
$$

and then, we conclude for the global asymptotic stability of both $z(t)$ and $\bar{k}(t)$. 
ii. If $I \in\left(-\frac{\alpha^{2}}{4 \beta}, L_{0}\left(\beta L_{0}-\alpha\right)\right)$, we have that $L\left(t^{*}\right)=0$ for a finite time $t^{*}$ given by:

$$
t^{*}=\frac{1}{b} \ln \left(\frac{\alpha-b}{\alpha+b} c\right) .
$$

It implies that $\lim _{t \rightarrow t^{*}} H(t)=+\infty$, and by (15), that $\lim _{t \rightarrow t^{*}} z(t)=$ $+\infty$. Then, $t=t^{*}$ is a vertical asymptote for both $z(t)$ and $\bar{k}(t)$.

Proposition 2. The steady-state capital per capita is given by $\bar{k}_{\infty}=\left(\frac{s A}{\delta}\right)^{\frac{1}{1-\varphi}}$, if $I \in\left[L_{0}\left(\beta L_{0}-\alpha\right),+\infty\right)$.

Proof. First, observe that we can find the horizontal asymptote $z_{\infty}$ for $z(t)$ making $\dot{z}=0$ in the differential equation (13), isolating $z(t)$, and taking its limit as $t \rightarrow+\infty$ :

$$
z_{\infty}=\lim _{t \rightarrow+\infty} \frac{s A}{\delta+\bar{n}(t)}
$$

where $\bar{n}(t)$, considering (8), is given by

$$
\bar{n}(t)=\frac{\dot{L}}{L}=\alpha-\beta L+\frac{I}{L}
$$

what implies that

$$
\bar{n}_{\infty}=\lim _{t \rightarrow+\infty} \bar{n}(t)=\frac{\alpha-b}{2}+2 \frac{\beta I}{\alpha+b}
$$

where we used the carrying capacity (11). Therefore, by (12), $\bar{k}_{\infty}$ can be written as:

$$
\bar{k}_{\infty}=\left(\frac{s A}{\delta+\bar{n}^{\infty}}\right)^{\frac{1}{1-\varphi}} .
$$

For $I \in\left[L_{0}\left(\beta L_{0}-\alpha\right),+\infty\right)$, we have that $\bar{n}^{\infty}=0$ by (17), and then:

$$
\bar{k}_{\infty}=\left(\frac{s A}{\delta}\right)^{\frac{1}{1-\varphi}} .
$$

Using (1), and the above results, we have the following similar results involving the output per capita $\bar{y}(t)$.

\section{Proposition 3.}

i. The output per capita $\bar{y}(t)$ is globally asymptotically stable for $I \in\left[L_{0}\left(\beta L_{0}-\alpha\right),+\infty\right)$.

ii. The output per capita goes to infinity at a finite time if $I \in\left(-\frac{\alpha^{2}}{4 \beta}, L_{0}\left(\beta L_{0}-\alpha\right)\right)$, that is, $\lim _{t \rightarrow t^{*}} \bar{y}(t)=+\infty$ where $t^{*}=\frac{1}{b} \ln \left(\frac{\alpha-b}{\alpha+b} c\right)$.

Proposition 4. The steady-state output per capita $\bar{y}_{\infty}$ is given by:

$\bar{y}_{\infty}=A\left(\frac{s A}{\delta}\right)^{\frac{\varphi}{1-\varphi}}$, if $I \in\left[L_{0}\left(\beta L_{0}-\alpha\right),+\infty\right)$.
Comparing the steady-states given by propositions 2(ii) and 4(ii), with the results obtained by Mingari Scarpello e Ritelli (2003) when $I=0, k_{\infty}$ and $y_{\infty}$, we conclude that:

$$
\bar{k}_{\infty}=k_{\infty} \text { and } \bar{y}_{\infty}=y_{\infty}
$$

\subsection{Closed-form Solution for $I>-\frac{\alpha^{2}}{4 \beta}$}

In order to solve the differential equation (5), we plug the modified population law (9) in (6), obtaining:

$$
\bar{k}(t)=\left\{e^{H(t)}\left[k_{0}^{1-\varphi}+(1-\varphi) s A \Im(t)\right]\right\}^{\frac{1}{1-\varphi}}
$$

where, by (14):

$$
\begin{aligned}
H(t) & =(\varphi-1) \int_{0}^{t}(\delta+n(\tau)) \mathrm{d} \tau \\
& =(\varphi-1) \delta t+\ln \left[\left(\frac{L(t)}{L_{0}}\right)^{\varphi-1}\right],
\end{aligned}
$$

and:

$$
\begin{aligned}
\Im(t) & =\int_{0}^{t} e^{-H(\tau)} \mathrm{d} \tau \\
& =\left(\frac{2 \beta L_{0}}{\alpha+b}\right)^{\varphi-1} \int_{0}^{t} e^{(1-\varphi) \delta \tau}\left(1-c e^{-b \tau}\right)^{\varphi-1} \times \\
& \times\left(1-\frac{\alpha-b}{\alpha+b} c e^{-b \tau}\right)^{1-\varphi} \mathrm{d} \tau .
\end{aligned}
$$

Defining $p=e^{-b \tau}$, we can rewrite $\mathfrak{I}(t)$ as:

$$
\begin{aligned}
& \mathfrak{I}(t)=-\frac{1}{b}\left(\frac{2 \beta L_{0}}{\alpha+b}\right)^{\varphi-1} \times \\
& \quad \times \int_{1}^{e^{-b t}} p^{\frac{(\varphi-1) \delta}{b}-1}(1-c p)^{\varphi-1}\left(1-\frac{\alpha-b}{\alpha+b} c p\right)^{1-\varphi} \mathrm{d} p,
\end{aligned}
$$

which can be written as:

$$
\mathfrak{I}(t)=-\frac{1}{b}\left(\frac{2 \beta L_{0}}{\alpha+b}\right)^{\varphi-1}\left[\mathcal{J}_{t}-\mathcal{J}_{0}\right],
$$

where $\mathcal{J}_{0}$ and $\mathcal{J}_{t}$ can be expressed in terms of the Beta and $F_{1}$ Appell functions:

$$
\begin{aligned}
& \mathcal{J}_{0}=\int_{0}^{1} p^{\frac{(\varphi-1) \delta}{b}-1}(1-c p)^{\varphi-1}\left(1-\frac{\alpha-b}{\alpha+b} c p\right)^{1-\varphi} \mathrm{d} p \\
& =\mathcal{B}\left(\frac{(\varphi-1) \delta}{b}, 1\right) \mathrm{F}_{1}\left(\begin{array}{c|c}
\frac{(\varphi-1) \delta}{b} ; 1-\varphi, \varphi-1 \\
\frac{(\varphi-1) \delta}{b}+1
\end{array} \mid c, \frac{\alpha-b}{\alpha+b} c\right),
\end{aligned}
$$

and

$$
\begin{aligned}
& \mathcal{J}_{t}=\int_{0}^{e^{-b t}} p^{\frac{(\varphi-1) \delta}{b}-1}(1-c p)^{\varphi-1}\left(1-\frac{\alpha-b}{\alpha+b} c p\right)^{1-\varphi} \mathrm{d} p \\
& =e^{(1-\varphi) \delta t} \int_{0}^{1} r \frac{(\varphi-1) \delta}{b}-1 \\
& =e^{(1-\varphi) \delta t} \mathcal{B}\left(\frac{(\varphi-1) \delta}{b}, 1\right) \mathrm{F}_{1}\left(\begin{array}{c}
\frac{(\varphi-1) \delta}{b} ; 1-\varphi, \varphi-1 \\
\frac{(\varphi-1) \delta}{b}+1
\end{array} \mid c e^{-b t}, \frac{\alpha-b}{\alpha+b} c e^{-b t}\right) .
\end{aligned}
$$


In calculations involving $\mathcal{J}_{t}$ we carried out the second change of variable $r=e^{b t} p$. Observe that, by $(22,23$, $24)$, from now on we must have $b>0$, which means, by (10), that the migration rate must satisfy the inequality $I>-\frac{\alpha^{2}}{4 \beta}$.

The Beta function $\mathcal{B}(a, b)$ is defined as Erdélyi (1953):

$$
\begin{aligned}
\mathcal{B}(a, b) & =\int_{0}^{1} u^{a-1}(1-u)^{b-1} d u \\
& =\frac{\Gamma(a) \Gamma(b)}{\Gamma(a+b)}, \operatorname{Re}(a), \operatorname{Re}(b)>0,
\end{aligned}
$$

and the $F_{1}$ Appell function is defined as the double hypergeometric series:

$$
\mathrm{F}_{1}\left(\begin{array}{c}
a ; a_{1}, a_{2} \\
a+b
\end{array} \mid z_{1}, z_{2}\right)=\sum_{n, k=0}^{\infty} \frac{(a)_{n+k}\left(a_{1}\right)_{k}\left(a_{2}\right)_{n}}{k ! n !(a+b)_{n+k}} z_{1}^{k} z_{2}^{n}
$$

which have the following integral representation (Slater, 1966):

$$
\mathrm{F}_{1}\left(\begin{array}{c}
a ; a_{1}, a_{2} \\
a+b
\end{array} \mid z_{1}, z_{2}\right)=\frac{1}{\mathcal{B}(a, b)} \int_{0}^{1} t^{a-1}\left(1-z_{1} t\right)^{-a_{1}}\left(1-z_{2} t\right)^{-a_{2}} d t
$$

under the conditions that $\operatorname{Re}(a), \operatorname{Re}(b)>0$ and $\left|z_{1}\right|,\left|z_{2}\right|<$ 1. This function is available in the Mathematica ${ }^{\circledR}$ CAS, a symbolic handling software we used in the simulations. Important to note that all results have been numerically validated, using a standard Euler Method implementation.

Finally, the closed-form solution of the logistic SolowSwan model with constant migration is given by (1) and $(21-24)$, where I may satisfy:

- $-\frac{\alpha^{2}}{4 \beta}<I<0$ : in this case we have emigration of labor force, situation that can occur in some specific labor market, as in the brain drain phenomena (Pieretti e Zou, 2009);

- $I=0$ : here the labor force growth is given by the pure logistic equation, and we recover the solution obtained in Mingari Scarpello e Ritelli (2003);

- $I>0$ : now we have immigration of labor force, i.e., the arriving of new workers from outside the economy, at a constant rate.

We highlight that under intense emigration $(I<0)$, the labor force (9) converge to zero at a finite time $t^{*}$, and consequently, the economy collapses $\left(Y\left(t^{*}\right)=0\right)$, by Proposition 1(ii) and (1). This will happen whenever $-\frac{\alpha^{2}}{4 \beta}<I<L_{0}(\beta L 0-\alpha)$, and the time of collapse will be:

$$
t^{*}=\frac{1}{b} \ln \left[\frac{(\alpha-b)}{(\alpha+b)} c\right]
$$

where $a, b$, and $c$ are given by (10).

\section{Simulations}

In the simulations below we assume the following set of parameters:

$$
\begin{gathered}
\alpha=0.2, \beta=0.01, \varphi=0.5, \delta=0.05, \\
s=0.06, A=1, \bar{k}_{0}=2, L_{0}=1
\end{gathered}
$$

which implies that $I$ must be greater than -1 for our solution to be valid, and $I \geq-0.19$ to have an asymptotically increasing labor force.

In Fig. 1 we plotted the economy's gross output for some values of $I$ around zero. As we can see, $I>0(I<$ 0 ) implies a higher (minor) production than without migration, i.e., $I=0$. This behaviour is a consequence of the impact of the constant migration term, I, in the growth of the labor force, as shown in Fig. 2. In Fig. 3 and 4 we can see the time evolution of the capital per capita, $\bar{k}(t)$, and of the output per capita, $\bar{y}(t)$.

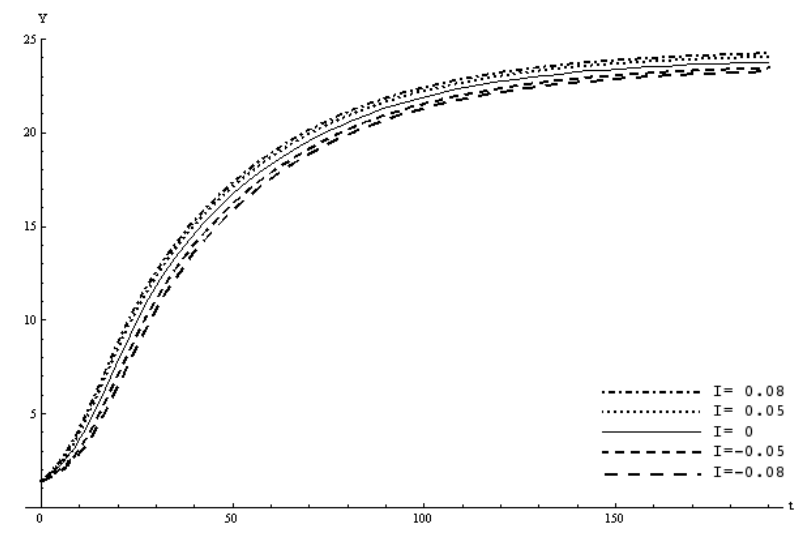

Figura 1: Gross Output versus Time

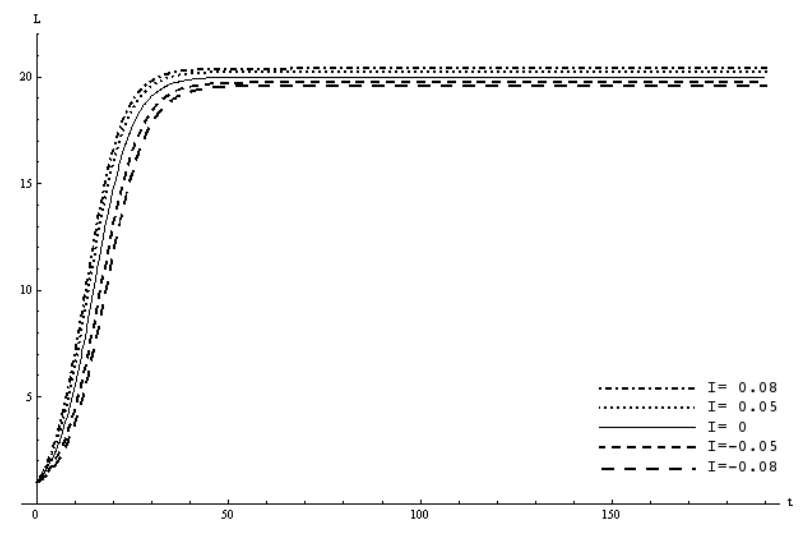

Figura 2: Labor Force versus Time 


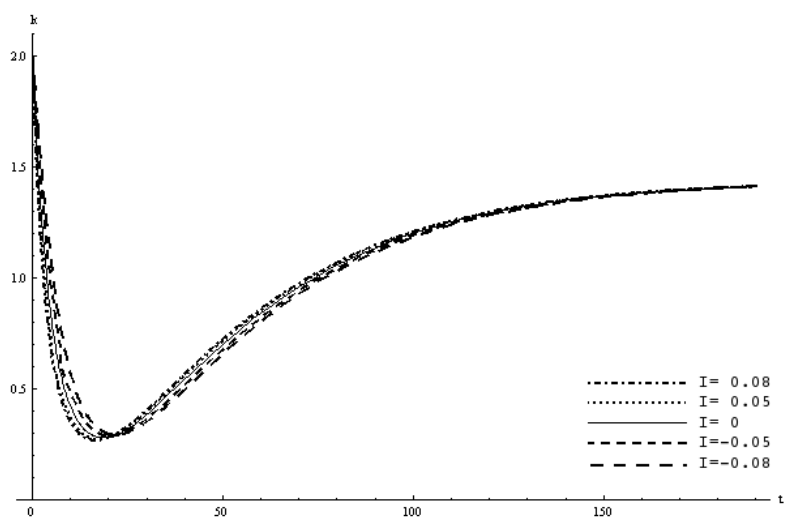

Figura 3: Capital per capita versus Time

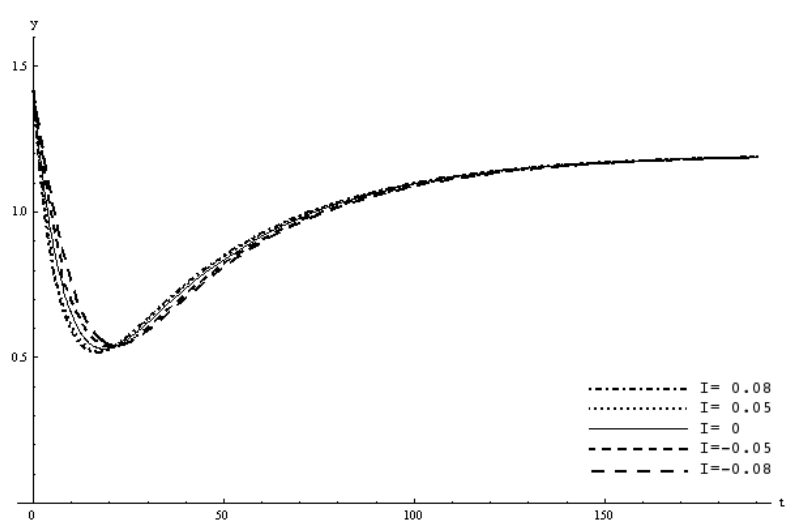

Figura 4: Output per capita versus Time

Note that in both cases:

- In the short term, the immigration of labor force $(I>0)$ implies a minor $\bar{k}(t)$ and $\bar{y}(t)$ than when there is no immigration $(I=0)$. Over the same period, the emigration $(I<0)$ implies a greater $\bar{k}(t)$ and $\bar{y}(t)$, when compared to $I=0$;

- In the middle term, the immigration of labor force $(I>0)$ implies a greater $\bar{k}(t)$ and $\bar{y}(t)$ than when there is no immigration $(I=0)$. Over the same period, the emigration $(I<0)$ implies a minor $\bar{k}(t)$ and $\bar{y}(t)$, when compared to $I=0$;

- In both cases, $\bar{k}(t)$ and $\bar{y}(t)$ converge to the same steady-state of the model with no migration, as $t \rightarrow \infty$ (see result (20)).

\section{Conclusions}

In this paper we derived a closed-form solution for the Solow-Swan model in terms of Beta and Appell $F_{1}$ special functions, considering the labor force growth ruled by a logistic equation added by a constant migra- tion rate, $I$. In this way, we extended the results obtained in Mingari Scarpello e Ritelli (2003). In addition, we proved the global asymptotic stability of $\bar{k}(t)$ and $\bar{y}(t)$, and showed that the output and capital per capita steady-states are equal to the model without migration: $\bar{k}_{\infty}=k_{\infty}$ and $\bar{y}_{\infty}=y_{\infty}$.

We also showed that our solution is valid for $I \geq$ $-\frac{\alpha^{2}}{4 \beta}$ (where $\alpha>0$ is the intrinsic growth rate, and $\beta>0$ is the crowding coefficient of the logistic equation), and that it converges to the Mingari Scarpello and Ritelli's solution when $I \rightarrow 0$.

Besides of that, we studied some transients for a set of parameters, and observed that, when compared to the case without migration $I=0$, immigration of labor force $(I>0)$ implies in a minor capital and production per capita, $\bar{k}(t)$ and $\bar{y}(t)$, in the short term and in greater values for $\bar{k}(t)$ and $\bar{y}(t)$ in the middle and long terms. In both cases, $\bar{k}(t)$ and $\bar{y}(t)$ convergence to the same steady-state given by the model without migration as $t \rightarrow \infty$. For $I<0$ (emigration of labor force), the inverse behaviour has been observed.

As a direct consequence of the labor force dynamics, we showed that the economy will collapse in finite time if the emigration is sufficiently intense, that is, if $-\frac{\alpha^{2}}{4 \beta}<$ $I<L_{0}\left(\beta L_{0}-\alpha\right)$.

\section{Acknowledgments}

\section{References}

Accinelli, E., Brida, J. G. (2005). Re-formulation of the solow economic growth model whit the richards population growth law. GE, Growth, Math methods 0508006, EconWPA, URL http://ideas.repec.org/p/wpa/wuwpge/0508006.html.

Banks, R. B. (1994). Growth and Diffusion Phenomena - Mathematical Frameworks and Applications. SpringerVerlag, USA.

Boucekkine, R., Ruiz-Tamarit, J. R. (2008). Special functions for the study of economic dynamics : The case of the lucas-uzawa model. Journal of Mathematical Economics, 44(1), 33-54.

Donghan, C. (1998). An improved solow-swan model. Chinese Quarterly Journal of Mathematics, 13(2), 72-78.

Erdélyi, A. (1953). Higher Transcendental Functions - Volume I. McGraw-Hill Book Company, USA.

Juchem Neto, J. P., Claeyssen, J. C. R. (2015a). Capitalinduced labor migration in a spatial solow model. Journal of Economics, 115(1), 25-47. 
Juchem Neto, J. P., Claeyssen, J. C. R., Ritelli, D., Mingari Scarpello, G. (2015b). Closed-form solution for the solow model with constant migration. Tendências em Matemática Aplicada e Computacional, 16(2), 1-13.

Maldonado, E. J. L., Brida, J. G. (2007). Closed form solutions to a generalization of the solow growth model. Applied Mathematical Sciences, 1(40), 1991-2000.

Matis, J. H., Kiffe, T. R. (2004). On stochastic logistic population growth models with immigration and multiple births. Theoretical Population Biology, 65(1), 89-104.

Mingari Scarpello, G., Ritelli, D. (2003). The solow model improved through the logistic manpower growth law. Annali Università di Ferrara - Sez VII - Sc Mat, 49(1), 73-83.

Pieretti, P., Zou, B. (2009). Brain drain and factor complementarity. Economic Modelling, 26(2), 404-413.

Rainville, E. D. (1960). Special Functions. The Macmillian Company, USA.

Slater, L. J. (1966). Generalized Hypergeometric Functions. Cambridge University Press, USA.

Solow, R. M. (1956). A contribution to the theory of economic growth. The Quarterly Journal of Economics, $70(1), 65-94$.

Swan, T. W. (1956). Economic growth and capital accumulation. Economic Record, 32, 334-361. 